\title{
Mucopolysaccharidosis type IIIB may predominantly present with an attenuated clinical phenotype
}

\author{
Marlies J. Valstar • Hennie T. Bruggenwirth • \\ Renske Olmer • Ron A. Wevers • Frans W. Verheijen • \\ Ben J. Poorthuis • Dicky J. Halley • Frits A. Wijburg
}

Received: 14 May 2010/Revised: 10 August 2010 /Accepted: 24 August 2010 /Published online: 18 September 2010

(C) The Author(s) 2010. This article is published with open access at Springerlink.com

\begin{abstract}
Mucopolysaccharidosis type IIIB (MPS IIIB, Sanfilippo syndrome type B) is a lysosomal storage disorder caused by deficiency of the enzyme $\mathrm{N}$-acetyl- $\alpha$ D-glucosaminidase (NAGLU). Information on the natural course of MPS IIIB is scarce but much needed in view of emerging therapies. To improve knowledge on the natural course, data on all 52 MPS IIIB patients ever identified by enzymatic studies in the Netherlands were gathered. Clinical data on 44 patients could be retrieved. Only a
\end{abstract}

Communicated by: Ed Wraith

Competing interest: None declared.

M. J. Valstar F. A. Wijburg

Department of Pediatrics and Amsterdam Lysosome Center

'Sphinx', Academic Medical Center, University of Amsterdam, Amsterdam, The Netherlands

H. T. Bruggenwirth $\cdot$ R. Olmer $\cdot$ F. W. Verheijen $\cdot$ D. J. Halley Department of Clinical Genetics, Erasmus University Medical Center,

Rotterdam, The Netherlands

R. A. Wevers

Laboratory of Genetic Endocrine and Metabolic Diseases,

University Medical Centre,

Nijmegen, The Netherlands

\section{B. J. Poorthuis}

Department of Medical Biochemistry and Amsterdam Lysosome Center 'Sphinx', Academic Medical Center, University of Amsterdam,

Amsterdam, The Netherlands

\section{F. A. Wijburg $(\bowtie)$}

Department of Pediatrics (G8-205), Academic Medical Center, Meibergdreef 9,

1105 AZ Amsterdam, The Netherlands

e-mail: f.a.wijburg@amc.uva.nl small number $(n=9 ; 21 \%)$ presented with a classical MPS III phenotype; all other patients showed a much more attenuated course of the disease characterized by a significantly slower regression of intellectual and motor abilities. The majority of patients lived well into adulthood. First signs of the disease, usually mild developmental delay, were observed at a median age of 4 years. Subsequently, patients showed a slowing and eventually a stagnation of development. Patients with the attenuated phenotype had a stable intellectual disability for many years. Molecular analysis was performed in 24 index patients. The missense changes p.R643C, p.S612G, p.E634K, and p.L497V were exclusively found in patients with the attenuated phenotype. MPS IIIB comprises a remarkably wide spectrum of disease severity, and an unselected cohort including all Dutch patients showed a large proportion (79\%) with an attenuated phenotype. MPS IIIB must be considered in patients with a developmental delay, even in the absence of a progressive decline in intellectual abilities. A key feature, necessitating metabolic studies, is the coexistence of behavioral problems.

\section{Introduction}

Mucopolysaccharidosis type III (MPS III, Sanfilippo syndrome) is an autosomal recessive lysosomal storage disorder caused by a deficiency of one of the enzymes involved in the degradation of the glycosaminoglycan heparan sulphate. Four different MPS III subtypes, MPS III types $\mathrm{A}, \mathrm{B}, \mathrm{C}$ and $\mathrm{D}$, are recognized based on the enzyme deficiency involved. Birth prevalences of 0.28 to 4.1 per 100,000 have been reported (Valstar et al. 2008).

Clinical signs, symptoms, and course of the disease are indistinguishable among the MPS III subtypes. The clinical 
course can generally be divided into three phases. After a symptom-free interval during the first months of life a slowing of mental development becomes apparent during the first phase of the disease, which usually starts between 1 and 4 years of age. This is followed by severe behavioral problems and progressive intellectual decline during the second phase which generally starts around 3-4 years. Finally, with the onset of severe dementia, the behavioral problems slowly disappear, and all motor functions start to decline, eventually resulting in complete loss of locomotion, dysphagia, and pyramidal tract lesions. Patients usually die at the end of the second or beginning of the third decade of life, although longer survival has been reported in patients with an attenuated phenotype (BergerPlantinga et al. 2004; van Schrojenstein-de Valk and van de Kamp 1987; Verhoeven et al. 2009).

MPS IIIB (Sanfilippo type B; MIM 252920) is caused by a deficiency of the enzyme N-acetyl- $\alpha$-D-glucosaminidase (NAGLU; E.C. 3.2.1.50), which hydrolyzes the $\alpha, 1 \rightarrow 4$ linkage between $\mathrm{N}$-acetylglucosamine and the neighboring uronic acid. The gene for human $\alpha-\mathrm{N}$ acetylglucosaminidase (NAGLU) was identified in 1996 and is localized on chromosome 17q21.1 (Zhao et al. 1996). The NAGLU cDNA encodes a 720 amino acid protein that has six potential N-glycosylation sites (Weber et al. 1996). Thus far, over 100 different mutations in the $N A G L U$ gene leading to MPS IIIB have been reported (Beesley et al. 1998, 2004, 2005; Bunge et al. 1999; Champion et al. 2010; Chinen et al. 2005; Coll et al. 2001; Emre et al. 2002; Esposito et al. 2000; Lee-Chen et al. 2002; Moog et al. 2007; Schmidtchen et al. 1998; Tessitore et al. 2000; Weber et al. 1999; Yogalingam and Hopwood 2001; Zhao et al. 1996, 1998). Most mutations are missense, but nonsense mutations, deletions, insertions, and splice-site mutations have also been reported.

Allele frequencies of the different mutations are very low, and most mutations are unique to individual families. This allelic heterogeneity likely causes the wide spectrum of clinical phenotypes observed in MPS IIIB (Weber et al. 1999), mainly characterized by a remarkable variability in the rate of progression (Moog et al. 2007; van de Kamp et al. 1981; van Schrojenstein-de Valk and van de Kamp 1987; Verhoeven et al. 2009). However, unbiased information on the natural course of MPS IIIB is limited.

Although no effective disease-modifying therapy is yet available for MPS IIIB, several studies on therapeutic interventions aimed at halting the devastating neurocognitive deterioration show promising results (Di et al. 2009; Piotrowska et al. 2006; Ponder and Haskins 2007). However, extensive knowledge on the natural course of the disorder as well as on potential genotype-phenotype correlations is needed to evaluate clinical efficacy of therapy.
In this study we report on the largest unbiased cohort of MPS IIIB patients to date, and we provide details on both the clinical history as well as on the molecular basis of disease, revealing large phenotypic variability and an association of the sequence variants p.R643C, p.S612G, p. E634K, and p.L497V with a significantly slower progression of the disease.

\section{Materials and methods}

\section{Recruitment of patients}

All patients with MPS IIIB ever diagnosed in the Netherlands were identified by retrieving the data from the combined registries of the four diagnostic centers for MPS III in the Netherlands (Poorthuis et al. 1999). Parents or legal representatives of patients were asked to participate in this study via the physicians who had requested the initial diagnostic studies. For deceased patients, we asked permission to study the medical records from the physician who had requested the initial diagnostic studies.

This study was approved by the Medical Ethical Committee of the AMC, Amsterdam.

\section{Medical history and clinical examination}

All living patients included in this study were seen at the outpatient clinic by one medical doctor (M.J.V.). A questionnaire on pregnancy and delivery, first clinical signs and symptoms, mental and motor milestones, behavioral problems, sleeping problems, and medical history was filled out by the parents or caregivers.

For deceased patients, medical records were retrieved, and all available relevant information was recorded.

\section{Cell culture}

Skin fibroblast cultures were obtained from the cell depository of the Department of Clinical Genetics, Erasmus Medical Centre in The Netherlands. The fibroblasts were cultured in Ham F10 medium (Lonza Biotech) supplemented with 10\% fetal calf serum. The fibroblasts were harvested by trypsinization and stored as cell pellet at $-80^{\circ} \mathrm{C}$.

DNA isolation and sequencing

DNA from the deceased patients was extracted from fibroblasts using the PureGene kit. DNA extracted from blood was isolated using Magnetic Separation Module 1 from Chemagen (Baesweiler, Germany).

Genomic DNA was used as a template for the PCR. The primers were designed using standard software (Primer3). 
All coding exons and exon/intron boundaries of the $N A G L U$ gene were sequenced. PCR products were purified using ExoSap-it (USB). Sequencing reactions were performed using the Big Dye Terminator kit according to the suppliers's instructions (Applied Biosystems). PCR products were purified with Performa V3 96-well short plates and analyzed using an ABI $3730 \mathrm{XL}$ analyzer (Applied Biosystems). The data were analyzed using SeqScape software (Applied Biosystems).

\section{Statistical analysis}

Data were analyzed using SPSS version 16.02 for windows. Descriptive statistics and frequencies were used for all calculations.

\section{Results}

Patients

In the registry of the Dutch diagnostic centers, records from 52 patients with MPS IIIB from 32 families were present (Table 1). All had been diagnosed between 1969 and 2008 on the basis of enzymatic studies.

Twenty-six of these 52 patients were alive at the time of this study and 24 patients could be included for full clinical studies. For two patients (patients 8.1 and 9.1; Table 1), clinical data were available until the ages of 38 and 10 , respectively, after which they were lost to follow-up. Twenty patients of the 52 were deceased at the time of the study. For 18 of them, extensive information on the course of the disease could be retrieved. In total, clinical data for 44 patients were available (Fig. 1).

Mutational analysis was performed in 24 of the 32 families.

Twenty-two patients from 12 families were previously reported in studies on MPS IIIB (Moog et al. 2007; van de Kamp et al. 1976; van Schrojenstein-de Valk and van de Kamp 1987; Verhoeven et al. 2009). Family \#19 consists of two closely related sibships (the fathers of these two sibships are brothers and the mothers are sisters).

Pregnancy and delivery

Pregnancy and delivery were without remarkable complications. All patients were born at term, and birth weights were all within normal range.

First signs and symptoms and diagnosis

After a symptom-free interval, patients presented at a median age of 4 years (range 1-10 years). The main symptom at presentation, seen in the majority of patients (95\%), was developmental delay, frequently in combination with behavioral problems. Two patients were diagnosed because of hepatomegaly.

The definitive diagnosis of MPS IIIB in the index patients was made at a median age of 13 years (range 1-66 years)

Behavioral and sleeping problems

With the exception of three patients (aged 2, 20, and 27 years at the time of the study), all patients were reported to have behavioral problems. Behavioral problems were first noted at a median age of 5 years (range 1-44), and usually consisted of extreme restlessness. In addition, temper tantrums, crying fits, and aggressive and destructive behavior were reported. Aggressive behavior was experienced as extremely problematic in several older patients, due to their preserved motor functions and physical strength.

Difficulties in falling asleep and/or frequent nocturnal wakening were reported in $63 \%$ of patients. Behavioral problems as well as sleeping problems were frequently episodic with periods of improved to even normal behavior and sleeping patterns.

Hearing and vision

Reliable assessment of vision and hearing is generally not feasible in MPS III patients because of behavioral problems and intellectual disability. In most patients hearing was considered to be (near) normal. Reliable audiometric testing was performed in 10 patients and revealed hearing problems in only 2. Visual acuity was measured in 17 patients and was without abnormalities in 9 patients. Retinitis pigmentosa was detected in 6 patients, all examined after the age of 40 years.

\section{Epilepsy}

Twenty-two of the 43 patients for whom data were available developed epilepsy during the course of the disease. Age of development of epilepsy is shown in Fig. 2. Patient 18.1, not included as an epileptic patient, was diagnosed with infantile spasms before the age of 2 years but has been seizure free since. Epilepsy could easily be controlled in the majority of patients.

Miscellaneous signs and symptoms

The majority of patients $(68 \%)$ suffered from recurrent ENT infections during the first years of life. Diarrhea, usually occurring in episodes, was reported in $70 \%$ of patients. Constipation frequently occurred in older patients. 
Table 1 Patients

\begin{tabular}{|c|c|c|c|c|c|c|}
\hline Family & $\mathrm{M} / \mathrm{F}$ & Birth year & $\begin{array}{l}\text { Age at time of study or } \\
\text { death (years) }\end{array}$ & Mutation 1 & Mutation 2 & Family connections \\
\hline 1.1 & $\mathrm{~F}$ & 1972 & 28 & p.R643C & p.R643C & \\
\hline 2.1 & M & 1975 & $\#$ & p.R297X & \#\# & \\
\hline 3.1 & $\mathrm{~F}$ & 1979 & \# & No DNA & & \\
\hline 4.1 & $\mathrm{~F}$ & 1988 & 20 & p.A72_G79dup8 & p.A72_G79dup8 & Sister patient 4.2 \\
\hline 4.2 & M & 1991 & 17 & p.A72_G79dup8 & p.A72_G79dup8 & Brother patient 4.1 \\
\hline 5.1 & $\mathrm{M}$ & 1952 & $44 \dagger$ & No DNA & & \\
\hline 6.1 & $\mathrm{~F}$ & 1932 & $69 \dagger$ & No DNA & & \\
\hline 7.1 & $\mathrm{~F}$ & 1952 & 58 & p.H248R & \#\# & \\
\hline 8.1 & M & 1968 & $38 \#$ & p.R643C & p.R643C & \\
\hline 9.1 & M & 1988 & $10 \#$ & No DNA & & \\
\hline 10.1 & M & 1942 & 67 & p.S612G & p.R177W & Brother patient 10.2 \\
\hline 10.2 & $\mathrm{~F}$ & 1950 & 58 & p.S612G & p.R177W & Sister patient 10.1 \\
\hline 11.1 & M & 1963 & $14 \dagger$ & p.R297X & p.R297X & \\
\hline 12.1 & $\mathrm{~F}$ & 1981 & 27 & p.R643C & p.R643C & \\
\hline 13.1 & $\mathrm{~F}$ & 1949 & 59 & p.D63N & \#\# & Aunt patient 14.1 \\
\hline 14.1 & M & 1983 & 25 & p.D63N & \#\# & Cousin patient 13.1 \\
\hline 15.1 & $\mathrm{~F}$ & 1989 & 19 & p.R565W & p.E634K & \\
\hline 15.2 & $\mathrm{~F}$ & 1988 & 20 & p.R565W & p.E634K & \\
\hline 16.1 & $\mathrm{~F}$ & 1953 & $45 \dagger$ & p.L497V & p.P521L & Sib family 16 \\
\hline 16.2 & M & 1959 & 50 & p.L497V & p.P521L & Sib family 16 \\
\hline 16.3 & $\mathrm{~F}$ & 1942 & $53 \dagger$ & p.L497V & p.P521L & Sib family 16 \\
\hline 16.4 & M & 1951 & $50 \dagger$ & p.L497V & p.P521L & Sib family 16 \\
\hline 16.5 & M & 1943 & 65 & p.L497V & p.P521L & Sib family 16 \\
\hline 16.6 & M & 1955 & 54 & p.L497V & p.P521L & Sib family 16 \\
\hline 17.1 & M & 1965 & $22 \dagger$ & p.R674H & p.R674H & \\
\hline 18.1 & M & 1984 & 25 & p.A282V & p.Y391C & \\
\hline 19.1 & $\mathrm{~F}$ & 1947 & 62 & p.R643C & p.R643C & Sib 19.2 , cousin $19.3,19.4,19.5,19.6$ \\
\hline 19.2 & $\mathrm{~F}$ & 1953 & 56 & p.R643C & p.R643C & Sib 19.1 , cousin $19.3,19.4,19.5,19.6$ \\
\hline 19.3 & $\mathrm{~F}$ & 1948 & $28 \dagger$ & p.R643C & p.R643C & Sib $19.4,19.5,19.6$, cousin $19.1,19.2$ \\
\hline 19.4 & M & 1951 & $51 \dagger$ & p.R643C & p.R643C & Sib $19.3,19.5,19.6$, cousin $19.1,19.2$ \\
\hline 19.5 & $\mathrm{~F}$ & 1954 & 54 & p.R643C & p.R643C & Sib $19.3,19.4,19.6$, cousin $19.1,19.2$ \\
\hline 19.6 & $\mathrm{~F}$ & 1956 & $52 \dagger$ & p.R643C & p.R643C & Sib $19.3,19.4,19.5$, cousin $19.1,19.2$ \\
\hline 20.1 & M & 1969 & $14 \dagger$ & p.R297X & p.V75fs & Brother patient 21.2 \\
\hline 20.2 & $\mathrm{~F}$ & 1971 & $13 \dagger$ & p.R297X & p.V75fs & Sister patient 21.1 \\
\hline 21.1 & $\mathrm{~F}$ & 1975 & $\#$ & No DNA & & Sister patient 22.1 \\
\hline 21.2 & $\mathrm{~F}$ & 1982 & $\#$ & No DNA & & Sister patient 22.1 \\
\hline 22.1 & $\mathrm{~F}$ & 1972 & $20 \dagger$ & \#\# & \#\# & \\
\hline 23.1 & M & 1976 & \# & No DNA & & Brother patient 24.2 \\
\hline 23.2 & $\mathrm{~F}$ & 1978 & $\#$ & No DNA & & Sister patient 24.1 \\
\hline 24.1 & $\mathrm{M}$ & 1973 & 35 & p.R565Q & p.A72_G79dup8 & Brother patient 25.2 \\
\hline 24.2 & M & 1971 & $29 \dagger$ & p.R565Q & p.A72_G79dup8 & Brother patient 25.1 \\
\hline 25.1 & M & 1997 & 11 & p.S612G & p.S612G & \\
\hline 26.1 & $\mathrm{~F}$ & 2004 & 4 & p.I403T & p.A72_G79del8 & \\
\hline 27.1 & $\mathrm{~F}$ & 1991 & $17 \dagger$ & No DNA & & \\
\hline 28.1 & M & 1961 & 48 & p.R297X & p.S612G & Brother patient 29.2 \\
\hline 28.2 & M & 1967 & $43 \dagger$ & p.R297X & p.S612G & Brother patient 29.1 \\
\hline 29.1 & $\mathrm{M}$ & 1988 & 20 & No DNA & & \\
\hline 30.1 & M & 2005 & 2 & p.R297X & p.S612G & \\
\hline 31.1 & $\mathrm{~F}$ & 1937 & $47 \dagger$ & p.R643C & p.R94_D95delinsPH & Sister patient 32.2 \\
\hline 31.2 & $\mathrm{M}$ & 1942 & $50 \dagger$ & p.R643C & p.R94_D95delinsPH & Brother patient 32.1 \\
\hline 32.1 & $\mathrm{M}$ & 1962 & $\# \dagger$ & p.R297X & p.R676P & Brother patient 33.2 \\
\hline 32.2 & $\mathrm{~F}$ & 1966 & $\# \dagger$ & p.R297X & p.R676P & Sister patient 33.1 \\
\hline
\end{tabular}

\# Lost to follow-up,

$\dagger$ deceased,

\#\# mutation not identified 
Fig. 1 Flow diagram of MPS IIIB patients included in the study

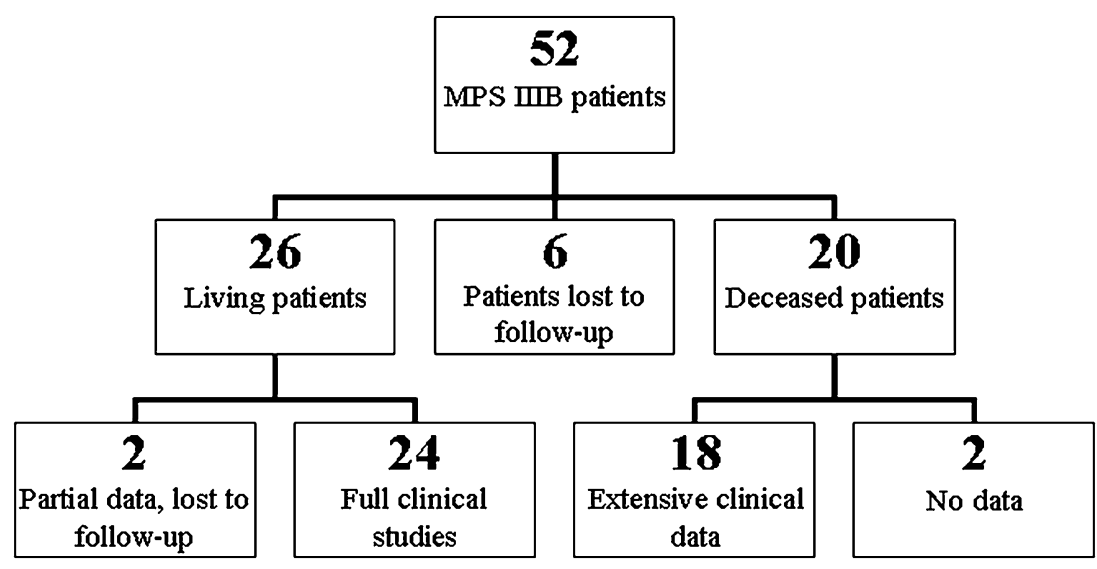

Cardiac abnormalities, usually consisting of mild valvular leakage, were reported in 10 patients. Two patients showed left ventricular hypertrophy in combination with severe stenosis of the aortic valve. Two other patients, both above the age of 50, had atrial fibrillation.

Hepatosplenomegaly was found in the minority of patients. In eight patients hepatomegaly was reported, while splenomegaly was only found in three patients. Hernias were found in 13 patients: 5 patients had suffered from an umbilical hernia and 10 patients from an inguinal hernia.

Age at death and cause of death

Eighteen of the 52 patients were deceased at the time of the study. They had died at a median age of 43.5 years (range 13-69). Information on the cause of death was available for 17 patients. The most common cause of death was pneumonia $(n=7)$. Other causes of death were withdrawal of artificial feeding because of profound functional disabilities $(n=2)$, accidental strangulation in restraints $(n=1)$, pancreatitis $(n=1)$, surgical complications $(n=1)$, aspiration of blood during a severe nasal bleeding $(n=1)$, and heart failure $(n=1)$. Three patients died suddenly without a known cause. Two of these patients were unexpectedly found dead in their bed at age 17 and 46 years and one

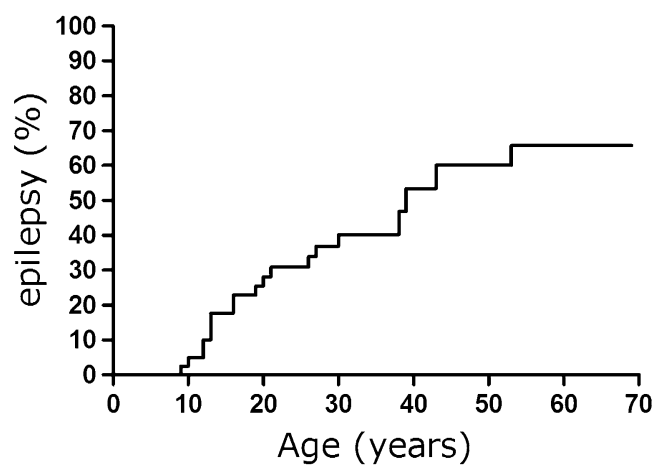

Fig. 2 Hazard curve of epilepsy in MPS IIIB patients $(n=43)$ patient died suddenly after an oral feeding at the age of 46 years. All three patients were completely bedridden, and all three had epilepsy that was well controlled by antiepileptic drugs. No immediate cause of death was detected in these three patients. Post mortem studies were performed in only one (aged 46), also failing to reveal the cause of death.

Molecular analyses

DNA was available for sequencing of the $N A G L U$ gene in 24 of the 32 MPS IIIB families. In 20 families, two mutations could be identified. In three families, we were able to detect only one mutation (families \#7, 13, and 14). In patient 22.1 no mutations were identified. Nineteen different sequence variants were identified, including 4 insertions, 1 deletion, 13 missense mutations, and 1 nonsense mutation (Table 2). Segregation was verified in patients 12.1, 25.1, 26.1, and 30.1 (Table 1).

\section{Course of the disease}

A remarkably large variability in the course of the disease was detected with an unusually mild course in the majority of patients (Fig. 3). Clinical phenotype could be assessed in 42 of the 44 patients for whom clinical data were available, since two patients, 2 and 4 years old at the time of the study, were excluded since no reliable phenotype could yet be assessed. These two patients were identified on the basis of hepatomegaly and developmental delay, respectively.

Severe phenotype

Patients with the classical severe phenotype of MPS III are generally fully dependent on external care, wheelchair dependent as early as their teenage years, and usually die within the first two decades of life. This severe phenotype was present in the minority $(n=9 ; 21 \%)$ of patients in our cohort (Patients 4.1, 4.2, 9.1, 11.1, 17.1, 20.1, 20.2, 22.1, 
Table 2 Sequence variants

\begin{tabular}{|c|c|c|}
\hline Nucleotide change & Amino acid change & Reference \\
\hline c. $187 \mathrm{G}>\mathrm{A}$ & p.D63N & Not previously reported \\
\hline c.214_237del24 & p.A72_G79del8 & Not previously reported \\
\hline c.214_237dup24 & p.A72_G79dup8 & Bunge et al. 1999 (published as c.238ins24) \\
\hline c.217_221dup5 & p.V75fs & Tessitore et al. 2000 \\
\hline c.281_283delinsCCC & p.R94_D95delinsPH & Not previously reported \\
\hline c. $529 \mathrm{C}>\mathrm{T}$ & p.R177W & Verhoeven et al. 2009 \\
\hline c. $743 \mathrm{~A}>\mathrm{G}$ & p.H248R & Weber et al. 1999 \\
\hline c. $845 \mathrm{C}>\mathrm{T}$ & p.A282V & Not previously reported \\
\hline c. $1172 \mathrm{~A}>\mathrm{G}$ & p.Y391C & Not previously reported \\
\hline c. $889 \mathrm{C}>\mathrm{T}$ & p.R297X & Zhao et al. 1998, Weber et al. 1999 \\
\hline c. $1208 \mathrm{~T}>\mathrm{C}$ & p.I403T & Not previously reported \\
\hline c. $1489 \mathrm{C}>\mathrm{G}$ & p.L497V & Not previously reported \\
\hline c. $1562 \mathrm{C}>\mathrm{T}$ & p.P521L & Zhao et al. 1998, Beesley et al. 1998, Weber et al. 1999 \\
\hline c. $1693 \mathrm{C}>\mathrm{T}$ & p.R565W & Beesley et al. 1998, Weber et al. 1999 \\
\hline c. $1694 \mathrm{G}>\mathrm{A}$ & p.R565Q & Bunge et al. 1999 \\
\hline c. $1834 \mathrm{~A}>\mathrm{G}$ & p.S612G & Zhao et al. 1998 \\
\hline c. $1900 \mathrm{G}>\mathrm{A}$ & p.E634K & Not previously reported \\
\hline c. $1927 \mathrm{C}>\mathrm{T}$ & p.R643C & Weber et al. 1999 \\
\hline c. $2021 \mathrm{G}>\mathrm{A}$ & p.R674H & Zhao et al. 1998 \\
\hline c. $2027 \mathrm{G}>\mathrm{C}$ & p.R676P & Weber et al. 1999 \\
\hline
\end{tabular}

and 27.1; Table 1). The clinical course of the disease in these severely affected patients is shown in Fig. 3. First clinical signs in this group of patients, consisting of developmental delay, mainly in speech development, were observed at a median age of 3 years. Patients in this group lost their ability to speak at a median age of 7.5 years (range 5-10 years) and the ability to walk at the age of 12 years (range $8-18$ years).

DNA was available in seven out of nine of these patients from four families and revealed mutations in six. Four different pathogenic mutations were identified: two insertions [c.214_237dup24 (p.A72_G79dup8) and c.217_221dup5 (p.V75fs)], one nonsense mutation (p.R297X), and one missense mutation (p.R674H).

\section{Attenuated phenotype}

The remaining 33 patients of the 42 for whom data on the clinical phenotype were available showed an attenuated phenotype, characterized by a much slower regression of intellectual and motor abilities (Fig. 3). Almost all (32) of these patients are adults or lived into adulthood. First clinical signs in this group were observed at a median age of 4 years, when a mild developmental delay was observed in all patients, except for one patient who was diagnosed because of hepatomegaly. Subsequently, a slowing of intellectual development eventually followed by full stagnation of development was observed. These older patients were reported to be able to function with a stable intellectual disability for many years. Loss of ability to speak was reported at a median age of 35 years (range 8-68 years) and of the ability to walk at a median age of 42.5 years (range 18-68 years). Diagnosis is this group of patients was made at a median age of 28 years.

Twelve of these 33 patients with an attenuated phenotype descended from two large families (families \#16 and 19, Table 1). These 12 patients have been previously reported (Moog et al. 2007; van de Kamp et al. 1976, 1981; van Schrojenstein-de Valk and van de Kamp 1987).

The six patients from family \#19 were homozygous for the pathogenic mutation p.R643C. This mutation was also detected in homozygous form in patients 1.1, 8.1, and 12.1 and in combination with an insertion [c.281_283delinsCCC (p.R94_D95delinsPH), unclassified variant] in the two patients from family \#31. The six patients in family \#16 were compound heterozygous for the combination of the missense changes p.L497V and p.P521L.

The two brothers with MPS IIIB from family \#24 also showed an attenuated phenotype. One of the two brothers died at the age of 29 due to complications of pancreatitis. The other patient is currently alive at the age of 35 years. $\mathrm{He}$ is wheelchair bound, but makes social contact and is able to eat with assistance. These patients were found to be compound heterozygous for p.R565Q and c.214_237dup24 (p.A72_G79dup8). 

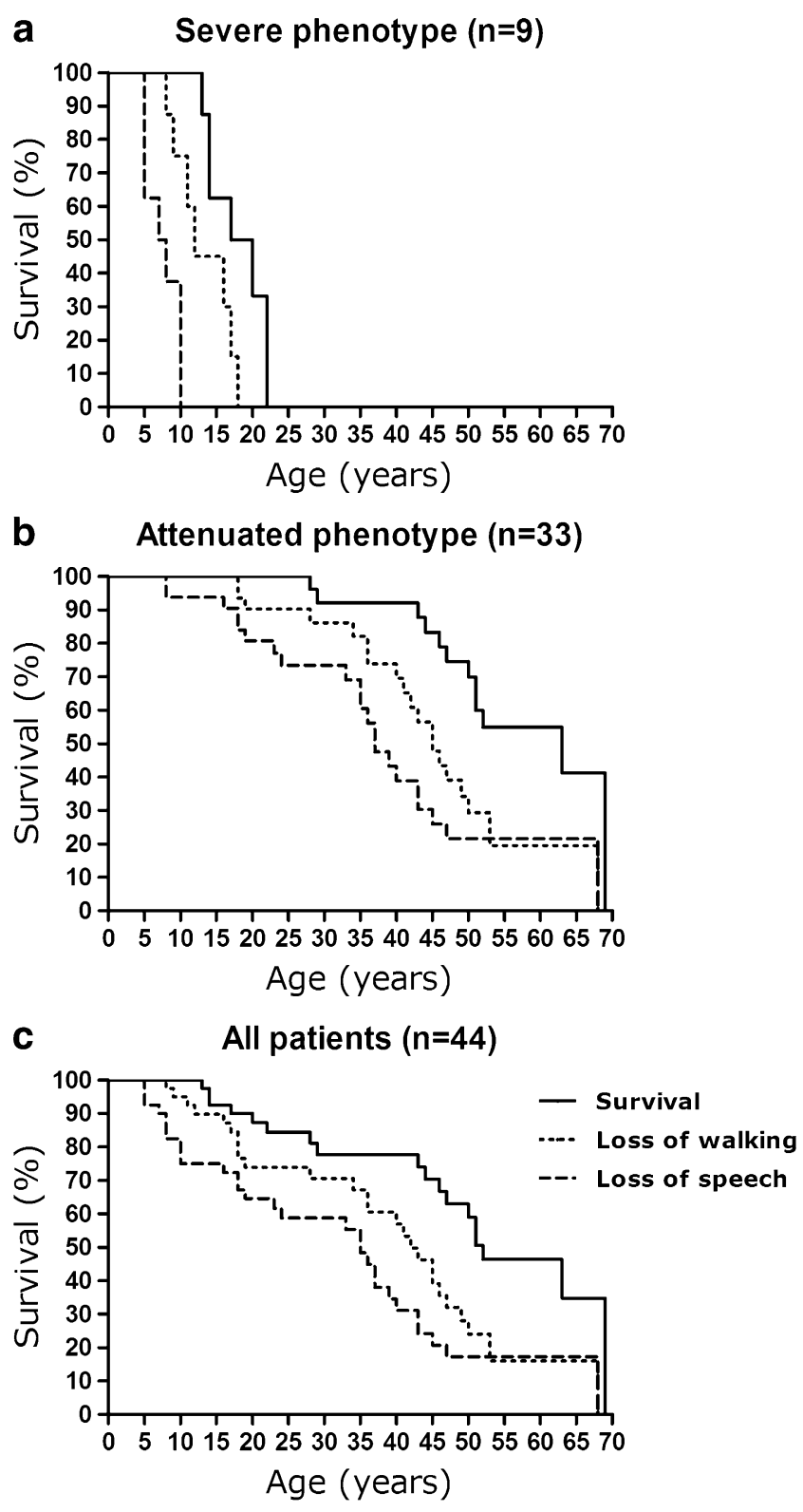

Fig. 3 Regression and survival in MPS IIIB patients of different phenotypes. Loss of walking indicates full loss of independent walking and loss of speech indicates full loss of speech

Finally, the missense change p.S612G was found in three families with an attenuated phenotype (families \#10, 25, and 28). Patients from family $\# 28$, who are compound heterozygous for the p.S612G and the pathogenic nonsense mutation p.R297X, showed an attenuated phenotype. One of these patients died at the age of 43 years due to pneumonia. His brother at the current age of 47 years showed a progressive intellectual decline from the age of 30 years, but he is still able to read and write simple words. His developmental age was 6 years (WISC-III) at the age of 47 years.

An 11-year-old boy was found to be homozygous for the missense change p.S612G. This patient shows an extremely mild MPS IIIB phenotype and is able to read and write and his intellectual functions still improve. Developmental testing (WISC-III) revealed an IQ of 53, corresponding with a developmental age of 6.5 years.

The remaining two patients (family \#10) have recently been reported (Verhoeven et al. 2009). These patients also show an extremely attenuated phenotype. The female patient (patient 10.2) is, as far as we know, the first woman with Sanfilippo syndrome to have both married and given birth to a child. Her brother (patient 10.1) is currently 67 years old. Developmental testing in this 67-year-old man revealed a developmental age of 2 years and 7 months (BSID-II). In this family, the unclassified variant p.S612G was found in heterozygous form, in combination with the novel missense change p.R177W.

\section{Discussion}

We report on the clinical and molecular characteristics of all patients ever diagnosed in the Netherlands with MPS IIIB and provide a complete and unbiased overview of the clinical course.

Patients included in this study follow the same general pattern of the disease progression as observed in patients with the Sanfilippo syndrome (Valstar et al. 2008). After an initial symptom-free interval, patients usually present with a slowing of developmental and/or behavioral problems, followed by progressive intellectual decline resulting in severe dementia and progressive motor disease.

The present study demonstrates a large variability in the course of the disease in patients with MPS IIIB, with an unusual mild course in the majority of patients. This was previously reported in other studies on Dutch patients with MPS IIIB (Moog et al. 2007; van de Kamp et al. 1976; Verhoeven et al. 2009). Patients with the classical severe phenotype of MPS III become completely dependent on external care and fully wheelchair dependent during their teenage years and usually die within the first two decades of life. This severe phenotype was present in only a minority of patients with MPS IIIB in this otherwise unbiased Dutch cohort $(n=9 ; 21 \%)$. Molecular analyses revealed two insertions [c.214_237dup24 (p.A72_G79dup8) and c.217_221dup5 (p.V75fs], one nonsense mutation (p. $\mathrm{R} 297 \mathrm{X}$ ), and a missense mutation (p.R674H) in this group of patients. Patients homozygous or compound heterozygous for a combination of insertions and nonsense mutation can be expected to have a severe phenotype. Several insertions in the $N A G L U$ gene have been reported previously in association with a severe phenotype. These insertions cause a change in reading frame resulting in altered full change in amino acid sequence and/or premature chain termination (Yogalingam and Hopwood 2001). 
The p.R297X mutation has been frequently reported in MPS IIIB patients (Yogalingam and Hopwood 2001), and all patients homozygous for this mutation were severely affected (Weber et al. 1999; Zhao et al. 1998). Finally the missense mutation p.R674H has also been reported previously in association with the severe MPS III phenotype (Zhao et al. 1998).

A remarkably large number of patients in our cohort showed an attenuated phenotype of the Sanfilippo syndrome, with long lasting stable intellectual disability with regression only following later in life and with prolonged survival. The pathogenic mutation p.R643C was found in five families, including four families with homozygous form and one family in combination with an insertion. The mutation p.R643C has previously been reported in association with an attenuated phenotype (Weber et al. 1999).

The missense changes p.L497V and p.P521L were detected in compound heterozygous form in family $\# 16$. The six patients from this family all showed an attenuated phenotype. The missense change p.P521L has been reported previously to be associated with a severe phenotype (Beesley et al. 1998; Weber et al. 1999; Zhao et al. 1998), suggesting that p.L497V probably conveys an attenuated phenotype. In the two sibs of family \#15, the missense changes p.E634K and p.R565W were identified in compound heterozygous form. The p.R565W change has been reported in association with a severe phenotype (Beesley et al. 1998; Weber et al. 1999), suggesting that the p.E634K missense change also conveys an attenuated phenotype. In vitro studies, expressing the p.L497V and p. E634K missense changes in cell lines are needed to demonstrate their functional consequences.

The p.S612G mutation, identified in three families (families \#10, 25, and 28), was also found to be associated with an attenuated phenotype. This was previously suggested by Zhao et al., who reported an attenuated patient who was compound heterozygous for $\mathrm{p} . \mathrm{S} 612 \mathrm{G}$ in combination with an insertion (Zhao et al. 1998). Our study reveals p.S612G in five patients with an attenuated phenotype: in homozygous form in one patient, in compound heterozygous form in combination with the severe mutation p.R297X in two patients, and in compound heterozygous form in combination with the novel missense change p.R177W in the remaining two patients, confirming the attenuated nature of the p.S612G mutation.

In conclusion we demonstrate that MPS IIIB conveys a remarkably broad phenotypic spectrum with over $70 \%$ of patients expressing an attenuated course of the disease in an otherwise unbiased Dutch cohort. This high percentage of patients with an attenuated form of MPS IIIB is remarkable and may be caused by a genetic bias. Indeed, 12 of the 33 patients with an attenuated phenotype reported in this study are from two large families (families \#16 and 19). However, another explanation might be a high degree of awareness of MPS III in the Netherlands leading to appropriate metabolic studies even in patients with a more attenuated phenotype. The studies on MPS III in the Netherlands by van der Kamp et al. (1976, 1981) were conducted in close collaboration with a large number of physicians throughout the country. This may have increased awareness of the phenotypic spectrum of MPS III in the Netherlands. The fact that 17 patients with an attenuated MPS IIIB phenotype from 14 families not related to any of the large MPS IIIB families were diagnosed within the last two decades supports this latter hypothesis.

Although the first clinical signs in these attenuated patients were observed already at a young age (median: 4 years), the diagnosis of MPS III was frequently made much later in life (median age at diagnosis: 28 years). These attenuated patients often functioned with a stable intellectual disability for many years, and in the majority of patients Sanfilippo syndrome was only considered as a possible cause of the disability after onset of a decline in intellectual capacities. Because of the need for appropriate genetic counselling as well as in view of emerging new therapies, MPS IIIB should therefore be included in the differential diagnosis in patients with a stable developmental delay, especially in combination with behavioral problems. Molecular analysis revealed 19 different sequence variants in this cohort, including a large number of unclassified variants. Functional in vitro assays will be needed to prove whether or not these novel variants are pathogenic.

Finally, we report that the missense changes p.R643C, p. S612G, p.E634K, and p.L497V in MPS IIIB are likely associated with an attenuated phenotype. Details on the natural course of the disease in combination with the genotype correlations may help in predicting the clinical course of the disease in newly diagnosed patients and may prove of essential importance in evaluating the effect of future therapies.

Acknowledgments This study was funded by grants from the Stichting Weeshuis der Doopsgezinden, Haarlem, the Netherlands, and Stichting Metakids, Amsterdam, the Netherlands. We thank the patients and their families and caretakers for participating in this study. We also thank all referring and collaborating physicians for giving us the opportunity to study their patients. Finally we would like to thank A. Schoonbrood, J.J.P. van de Kamp (†), and W. Onkenhout for helping us to contact attending physicians.

Open Access This article is distributed under the terms of the Creative Commons Attribution Noncommercial License which permits any noncommercial use, distribution, and reproduction in any medium, provided the original author(s) and source are credited. 


\section{References}

Beesley CE, Young EP, Vellodi A et al. (1998) Identification of 12 novel mutations in the alpha-N-acetylglucosaminidase gene in 14 patients with Sanfilippo syndrome type B (mucopolysaccharidosis type IIIB). J Med Genet 35:910-914

Beesley C, Moraitou M, Winchester B et al. (2004) Sanfilippo B syndrome: molecular defects in Greek patients. Clin Genet 65:143-149

Beesley CE, Jackson M, Young EP et al. (2005) Molecular defects in Sanfilippo syndrome type B (mucopolysaccharidosis IIIB). J Inherit Metab Dis 28:759-767

Berger-Plantinga EG, Vanneste JA, Groener JE et al. (2004) Adultonset dementia and retinitis pigmentosa due to mucopolysaccharidosis III-C in two sisters. J Neurol 251:479-481

Bunge S, Knigge A, Steglich C et al. (1999) Mucopolysaccharidosis type IIIB (Sanfilippo B): identification of 18 novel alpha$\mathrm{N}$-acetylglucosaminidase gene mutations. J Med Genet $36: 28-31$

Champion KJ, Basehore MJ, Wood T et al. (2010) Identification and characterization of a novel homozygous deletion in the alpha-Nacetylglucosaminidase gene in a patient with Sanfilippo type B syndrome (mucopolysaccharidosis IIIB). Mol Genet Metab 100:51-56

Chinen Y, Tohma T, Izumikawa Y et al. (2005) Sanfilippo type B syndrome: five patients with an R565P homozygous mutation in the alpha- $\mathrm{N}$-acetylglucosaminidase gene from the Okinawa islands in Japan. J Hum Genet 50:357-359

Coll MJ, Anton C, Chabas A (2001) Allelic heterogeneity in Spanish patients with Sanfilippo disease type B. Identification of eight new mutations. J Inherit Metab Dis 24:83-84

Di Domenico C, Villani GR, Di Napoli D et al. (2009) Intracranial gene delivery of LV-NAGLU vector corrects neuropathology in murine MPS IIIB. Am J Med Genet A 149A:1209-1218

Emre S, Terzioglu M, Tokatli A et al. (2002) Sanfilippo syndrome in Turkey: identification of novel mutations in subtypes A and B. Hum Mutat 19:184-185

Esposito S, Balzano N, Daniele A et al. (2000) Heparan N-sulfatase gene: two novel mutations and transient expression of 15 defects. Biochim Biophys Acta 1501:1-11

Lee-Chen GJ, Lin SP, Lin SZ et al. (2002) Identification and characterisation of mutations underlying Sanfilippo syndrome type B (mucopolysaccharidosis type IIIB). J Med Genet 39:E3

Moog U, van Mierlo I, van Schrojenstein Lantman-de Valk HM et al. (2007) Is Sanfilippo type B in your mind when you see adults with mental retardation and behavioral problems? Am J Med Genet C Semin Med Genet 145C:293-301

Piotrowska E, Jakobkiewicz-Banecka J, Baranska S et al. (2006) Genistein-mediated inhibition of glycosaminoglycan synthesis as a basis for gene expression-targeted isoflavone therapy for mucopolysaccharidoses. Eur J Hum Genet 14:846-852

Ponder KP, Haskins ME (2007) Gene therapy for mucopolysaccharidosis. Expert Opin Biol Ther 7:1333-1345

Poorthuis BJ, Wevers RA, Kleijer WJ et al. (1999) The frequency of lysosomal storage diseases in the Netherlands. Hum Genet 105:151-156

Schmidtchen A, Greenberg D, Zhao HG et al. (1998) NAGLU mutations underlying Sanfilippo syndrome type B. Am J Hum Genet 62:64-69

Tessitore A, Villani GR, Di Natale DC et al. (2000) Molecular defects in the alpha-N-acetylglucosaminidase gene in Italian Sanfilippo type B patients. Hum Genet 107:568-576

Valstar MJ, Ruijter GJ, van Diggelen OP et al. (2008) Sanfilippo syndrome: a mini-review. J Inherit Metab Dis 31:240-252

Van de Kamp JJ, van Pelt JF, Liem KO et al. (1976) Clinical variability in Sanfilippo B disease: a report on six patients in two related sibships. Clin Genet 10:279-284

Van de Kamp JJ, Niermeijer MF, von Figura FK et al. (1981) Genetic heterogeneity and clinical variability in the Sanfilippo syndrome (types A, B, and C). Clin Genet 20:152-160

Van Schrojenstein-de Valk HM, van de Kamp JJ (1987) Follow-up on seven adult patients with mild Sanfilippo B-disease. Am J Med Genet 28:125-129

Verhoeven WM, Csepan R, Marcelis CL et al. (2009) Sanfilippo B in an elderly female psychiatric patient: a rare but relevant diagnosis in presenile dementia. Acta Psychiatr Scand 122(2):162-165

Weber B, Blanch L, Clements PR et al. (1996) Cloning and expression of the gene involved in Sanfilippo B syndrome (mucopolysaccharidosis III B). Hum Mol Genet 5:771-777

Weber B, Guo XH, Kleijer WJ et al. (1999) Sanfilippo type B syndrome (mucopolysaccharidosis III B): allelic heterogeneity corresponds to the wide spectrum of clinical phenotypes. Eur J Hum Genet 7:34-44

Yogalingam G, Hopwood JJ (2001) Molecular genetics of mucopolysaccharidosis type IIIA and IIIB: diagnostic, clinical, and biological implications. Hum Mutat 18:264-281

Zhao HG, Li HH, Bach G et al. (1996) The molecular basis of Sanfilippo syndrome type B. Proc Natl Acad Sci USA 93:6101-6105

Zhao HG, Aronovich EL, Whitley CB (1998) Genotype-phenotype correspondence in Sanfilippo syndrome type B. Am J Hum Genet 62:53-63 